\title{
Sex-specific, male-line transgenerational responses in humans
}

\author{
Marcus E Pembrey ${ }^{* 1,2}$, Lars Olov Bygren ${ }^{3,6}$, Gunnar Kaati ${ }^{4}$, Sören Edvinsson ${ }^{5}$, \\ Kate Northstone ${ }^{2}$, Michael Sjöström ${ }^{6}$, Jean Golding ${ }^{2}$ and The ALSPAC Study Team ${ }^{2}$
}

${ }^{1}$ Clinical and Molecular Genetics Unit, Institute of Child Health, University College London, England, UK; ${ }^{2}$ Avon Longitudinal Study of Parents and Children, Bristol University, England, UK; ${ }^{3}$ Department of Community Medicine and Rehabilitation, Social Medicine, Umea University, Umeå, Sweden; ${ }^{4}$ Department of Public Health and Clinical Medicine, Umea University, Umea , Sweden; ${ }^{5}$ Demographic Database, Umea University, Umea, Sweden; ${ }^{6}$ Department of Biosciences, Preventive Nutrition Karolinska Institute, Karolinska, Sweden

Transgenerational effects of maternal nutrition or other environmental 'exposures' are well recognised, but the possibility of exposure in the male influencing development and health in the next generation(s) is rarely considered. However, historical associations of longevity with paternal ancestors' food supply in the slow growth period (SGP) in mid childhood have been reported. Using the Avon Longitudinal Study of Parents and Children (ALSPAC), we identified 166 fathers who reported starting smoking before age 11 years and compared the growth of their offspring with those with a later paternal onset of smoking, after correcting for confounders. We analysed food supply effects on offspring and grandchild mortality risk ratios (RR) using 303 probands and their 1818 parents and grandparents from the 1890, 1905 and 1920 Överkalix cohorts, northern Sweden. After appropriate adjustment, early paternal smoking is associated with greater body mass index (BMI) at 9 years in sons, but not daughters. Sex-specific effects were also shown in the Överkalix data; paternal grandfather's food supply was only linked to the mortality RR of grandsons, while paternal grandmother's food supply was only associated with the granddaughters' mortality RR. These transgenerational effects were observed with exposure during the SGP (both grandparents) or fetal/infant life (grandmothers) but not during either grandparent's puberty. We conclude that sex-specific, male-line transgenerational responses exist in humans and hypothesise that these transmissions are mediated by the sex chromosomes, $X$ and $Y$. Such responses add an entirely new dimension to the study of gene-environment interactions in development and health.

European Journal of Human Genetics (2006) 14, 159-166. doi:10.1038/sj.ejhg.5201538; published online 14 December 2005

Keywords: transgenerational responses; paternal ancestors; sex-specificity; slow growth period; body mass index; mortality

\section{Introduction}

Variation in people's response to nutritional or other environmental 'exposures' is determined by inherited

\footnotetext{
${ }^{*}$ Correspondence: Professor M Pembrey, Institute of Child Health, University College London, Picketts South, Picketts Lane, Salford, Redhill, Surrey RH1 5RG UK. Tel/fax: + 441293 771202;

E-mail: M.Pembrey@bristol.ac.uk

Received 10 June 2005; revised 1 September 2005; accepted 18 October 2005; published online 14 December 2005
}

differences, different developmental experiences or both. Inherited differences are traditionally regarded as the DNA sequence variations transmitted by parents. However, another possibility to be considered, in the gene-environment interplay underlying common disease, is the transgenerational response. This requires a mechanism for transmitting environmental exposure information that then alters gene expression in the next generation(s). There are opportunities for this down the female line through the egg cytoplasm or transplacental route. ${ }^{1}$ 
A transgenerational response down the male line implies the ancestral exposure information is carried by the sperm's chromosomes in some way, although the paucity of empirical observations suggesting such a transgenerational response means little mechanistic research has been performed. Viruses, prions, ${ }^{2}$ RNA molecules, ${ }^{3}$ 'responsive' DNA-sequences $^{4,5}$ or transmission of a particular epigenetic state could theoretically mediate transgenerational effects. Epigenetics refers to heritable changes in gene function that cannot be explained by changes in DNA sequence, with DNA methylation patterns being an important contribution to epigenetic memory. ${ }^{6}$ Murine studies have demonstrated transgenerational inheritance of epigenetic states at the agouti-viable yellow $\left(\mathrm{A}^{\mathrm{vy}}\right)$ allele and the Axin-fused $\left(\mathrm{Axin}{ }^{\mathrm{Fu}}\right)$ allele, the latter through both maternal and paternal transmission. ${ }^{7}$ A recent study on rats has shown the transgenerational action of endocrine disruptors on spermatogenic capacity together with an associated change in sperm DNA methylation. ${ }^{8}$

Earlier, we reported an association of ancestral food supply with longevity ${ }^{9}$ and with cardiovascular and diabetic mortality. ${ }^{10}$ These studies exploited historical records, including harvests and food prices, in Överkalix, an isolated community in northern Sweden. We demonstrated that the probands' longevity was influenced by their paternal grandfathers' access to food, but only during the grandfather's slow growth period (SGP) before the prepubertal peak in growth velocity and a period of relatively low need for food. ${ }^{9}$ Focusing on the SGP in a larger study of 1890,1905 and 1920 cohorts, ${ }^{10}$ the father's poor food supply and the mother's good food supply were associated with a lower risk of cardiovascular death. However, again there was also a striking association with the paternal grandfather's food supply, this time with his grandchild's risk of diabetic death. If these associations reflect (secondary) manifestations of a transgenerational response to nutritional or stress trends that evolved to maintain early survival and reproductive success, then early and prepubertal growth in the offspring would be outcomes of interest.

The present study was designed to detect a transgenerational effect through the male line in a contemporary population, the Avon Longitudinal Study of Parents and Children (ALSPAC). ${ }^{11}$ We wished to test the specificity of exposure in the SGP for triggering any such effect, this being a feature of the Swedish observations, and to note any pointers to underlying mechanisms such as sexspecific effects. In the absence of contemporary annual swings in food supply, we tested the ALSPAC fathers' SGP for cigarette-induced transgenerational effects on the growth of their future offspring and analysed the effect of ancestral food supply on mortality by sex of the proband using the Överkalix data.

\section{Materials and methods ALSPAC}

Recruited from all pregnancies in three districts around Bristol, UK, ALSPAC was designed with particular attention paid to early development. ${ }^{11}$ Women with due dates between 1.4.91 and 31.12.92 were enrolled as early in pregnancy as possible. The pregnant mother could invite her partner to take part. For 10000 of the 14024 pregnancies surviving 5 months gestation, a questionnaire was completed by the father. One question in mid-pregnancy was: 'Have you ever been a smoker?' 5451 of the 9886 fathers who answered this, responded positively; they were then asked 'at what age did you start smoking regularly?' All children were measured by the ALSPAC study team at birth, and at ages 7 and 9 years in the ALSPAC research clinic. ${ }^{12}$ Multiple regression analysis of the effect of paternal age at onset of smoking on mean body mass index (BMI), that is, weight $(\mathrm{kg}) /$ height $^{2}\left(\mathrm{~m}^{2}\right)$ allowed for parity (primiparae $v s$ multiparae), education, housing tenure, maternal smoking (yes vs no) and paternal smoking at conception (yes $v s$ no). $P$-values are two-tailed tests of $\mathrm{F}$ distribution and trend.

\section{Överkalix cohorts}

Tracing the probands born in the parish of Överkalix, northernmost Sweden and the use of harvest and food price records to assess food availability to the probands' parents and grandparents have been described elsewhere. ${ }^{9,10}$ In a $50 \%$ random sample comprising 320 people (probands) born in $1890(n=108), 1905$ $(n=99)$ and $1920(n=113)$ in Överkalix, three individuals could not be traced to their death, emigration or current residence and among 14 probands the birth year was missing for some of their six ancestors. This left us with 303 probands, 164 men and 139 women, and their 1818 parents and grandparents. Mortality follow-up stopped in 1995, when 44 were still alive. The ancestors' SGP had been set previously ${ }^{9}$ at 8-10 years for girls and 9-12 years for boys using modern cohort data ${ }^{13}$ adjusted for the secular decrease of age at puberty. ${ }^{14}$ The first analysis considered the six ancestorś exposure to: (a) good food supply during SGP, no year of poor supply but possibly years of moderate availability and (b) poor food supply during the SGP, no year of good supply but possibly years of moderate availability. For each sex of the probands the Cox proportional hazards model for survival was applied $^{15}$ to test and estimate the influence on the mortality of probands of each of the six ancestors' nutrition during his or her SGP, taking into account the other five ancestors' availability of food during their respective SGP and presented as risk ratios (RR). A second analysis explored the effect of paternal grandparental food supply at different times in their life up to the age of 20 years on the mortality risk ratio of their 
grandchildren, using 3-year rolling means for the proband's mortality RR.

\section{Results}

Paternal onset of smoking and BMI between 7 and 9 years

In the ALSPAC fathers who reported when they started smoking $(n=5357)$, the commonest age of onset was 16 years, but 166 reported smoking before 11 years of age when many would still be in their SGP. We therefore decided to compare the effect of the onset of paternal smoking at $<11$ years, 11-12 years, 13-14 years, $>14$ years, and of never smoking on BMI at 7 and 9 years. In an initial regression analysis, earlier onset of paternal smoking was associated with increased BMI in both girls and boys at 9 years, but this analysis had not adjusted for father's continued smoking up to conception. However, we found that the earlier the father started smoking the more likely he was to still be smoking at conception (data not shown). Correcting for this confounder, the association of age of paternal smoking onset with BMI at 9 years remained in boys (F distribution, $P=0.015$; trend, $P=0.025$ ), but disappeared in girls (Table 1 ).

\section{Sex-specific effects of ancestral food supply on mortality}

We looked for sex-specific effects in the Swedish data linking ancestral food supply to mortality using the three cohorts combined. Table 2 shows both parents' exposure to food availability during their SGP had significant influences on their daughter's mortality RR, with a possible effect of mother's food supply on her son's mortality RR. It should be noted that these parental findings are modified when the probands' own childhood circumstances are taken into account (see Discussion). There are no significant effects of SGP food availability for either maternal grandparent on the mortality RR in the grandchildren of either sex. However, on the paternal side there are significant effects on the grandchildren's relative mortality RR and they are strikingly sex-specific. The male (but not the female) probands had an increased $(P=0.009)$ mortality RR of 1.67 when the paternal grandfather had experienced good food supply during his SGP (compared to

Table 1 Adjusted mean (SE) BMI by age and sex against age of onset of paternal smoking

\begin{tabular}{|c|c|c|c|c|}
\hline \multirow[b]{2}{*}{ Age father started smoking } & \multicolumn{2}{|c|}{ Boys } & \multicolumn{2}{|c|}{ Girls } \\
\hline & Age 7 & Age 9 & Age 7 & Age 9 \\
\hline $\begin{array}{l}<10 \\
11-12 \\
13-14 \\
15+/ \text { never }\end{array}$ & $\begin{array}{l}16.53(0.33) \\
16.22(0.23) \\
16.07(0.14) \\
15.98(0.08)\end{array}$ & $\begin{array}{l}18.15(0.55) \\
17.73(0.35) \\
17.75(0.21) \\
17.23(0.12)\end{array}$ & $\begin{array}{l}16.45(0.37) \\
16.33(0.23) \\
16.54(0.16) \\
16.41(0.09)\end{array}$ & $\begin{array}{l}18.64(0.55) \\
18.22(0.32) \\
18.24(0.23) \\
18.04(0.12)\end{array}$ \\
\hline $\begin{array}{l}F(3 \mathrm{df}) \\
P \\
P \text { for trend }\end{array}$ & $\begin{array}{l}1.30 \\
0.271 \\
0.208\end{array}$ & $\begin{array}{l}3.49 \\
0.015 \\
0.025\end{array}$ & $\begin{array}{l}0.29 \\
0.833 \\
0.514\end{array}$ & $\begin{array}{l}0.64 \\
0.587 \\
0.173\end{array}$ \\
\hline
\end{tabular}

Adjusted for parity, education, maternal smoking, housing tenure and paternal smoking at conception.

Table 2 Mortality risk ratios of male and female probands depending on ancestors' food availability in their SGP; 1890,1905 and 1920 cohorts combined $(n=303)$

\begin{tabular}{|c|c|c|c|c|c|c|c|c|}
\hline & \multicolumn{8}{|c|}{$G_{o o d}{ }^{a}$ or poor food availability during SGP } \\
\hline & \multicolumn{4}{|c|}{ Good } & \multicolumn{4}{|c|}{ Poor } \\
\hline & \multicolumn{2}{|c|}{ Female probands } & \multicolumn{2}{|c|}{ Male probands } & \multicolumn{2}{|c|}{ Female probands } & \multicolumn{2}{|c|}{ Male probands } \\
\hline & $R R$ & $P$ & $R R$ & $P$ & $R R$ & $P$ & $R R$ & $P$ \\
\hline \multicolumn{9}{|l|}{ Ancestors exposed } \\
\hline Mother & 1.66 & 0.03 & 1.53 & 0.06 & 0.51 & 0.04 & 0.78 & 0.30 \\
\hline Father $^{\mathrm{c}}$ & 1.68 & 0.02 & 1.07 & 0.73 & 1.31 & 0.24 & 0.96 & 0.85 \\
\hline Maternal grandmother & 0.79 & 0.27 & 0.92 & 0.62 & 1.30 & 0.22 & 1.25 & 0.24 \\
\hline Maternal grandfather & 1.23 & 0.34 & 0.81 & 0.22 & 1.05 & 0.81 & 0.98 & 0.90 \\
\hline Paternal grandmother & 2.13 & 0.001 & 1.02 & 0.93 & 0.72 & 0.12 & 1.23 & 0.27 \\
\hline Paternal grandfather & 0.81 & 0.32 & 1.67 & 0.009 & 1.17 & 0.43 & 0.65 & 0.025 \\
\hline
\end{tabular}

${ }^{a}$ Good availability during SGP and no year of poor availability but possibly years of moderate availability.

${ }^{b}$ Poor availability during the SGP and no year of good availability but possibly years of moderate availability of food.

'These paternal effects are changed by adjustment for the proband's own childhood circumstances (see Discussion).

RR - mortality risk ratio. $P-P$-value.

Bold indicates significant results. 
Table 3 Effects of paternal grandparents' availability of food during SGP and their grandchildrens' mortality RR

\begin{tabular}{|c|c|c|c|c|c|c|c|c|}
\hline & \multicolumn{8}{|c|}{ Ancestors exposed } \\
\hline & \multicolumn{3}{|c|}{ Paternal grandfather } & & \multicolumn{4}{|c|}{ Paternal grandmother } \\
\hline & \multicolumn{2}{|c|}{$\operatorname{Good}^{a}$} & \multicolumn{2}{|c|}{ Poor ${ }^{b}$} & \multicolumn{2}{|c|}{$\operatorname{Good}^{\mathrm{a}}$} & \multicolumn{2}{|c|}{ Poor } \\
\hline & $R R$ & $P$ & $R R$ & $P$ & $R R$ & $P$ & $R R$ & $P$ \\
\hline \multicolumn{9}{|l|}{ Male probands } \\
\hline $1890(n=56)$ & 2.65 & 0.012 & 0.63 & 0.16 & 0.73 & 0.42 & 1.09 & 0.77 \\
\hline $1905(n=52)$ & 1.62 & 0.16 & 0.43 & 0.04 & 1.00 & 1.00 & 0.98 & 0.96 \\
\hline $1920(n=56)$ & 1.15 & 0.68 & 0.77 & 0.54 & 1.46 & 0.28 & 1.62 & 0.18 \\
\hline All 3 cohorts & 1.67 & 0.01 & 0.65 & 0.025 & 1.02 & 0.93 & 1.23 & 0.27 \\
\hline \multicolumn{9}{|l|}{ Female probands } \\
\hline $1890(n=50)$ & 0.94 & 0.87 & 1.22 & 0.56 & 2.25 & 0.01 & 0.62 & 0.16 \\
\hline $1905(n=42)$ & 1.00 & 1.00 & 0.88 & 0.73 & 2.82 & 0.01 & 0.39 & 0.03 \\
\hline $1920(n=47)$ & 1.090 & 0.849 & 1.54 & 0.38 & 1.093 & 0.848 & 0.93 & 0.89 \\
\hline All 3 cohorts & 0.81 & 0.32 & 1.17 & 0.43 & 2.13 & 0.001 & 0.72 & 0.12 \\
\hline
\end{tabular}

RR - mortality risk ratio. $P-P$-value.

${ }^{a}$ Good availability during SGP and no year of poor availability but possibly years of moderate availability.

${ }^{b}$ Poor availability during the SGP and no year of good availability but possibly years of moderate availability of food.

the risk for male probands whose paternal grandfather had other experiences during his SGP). The female (but not the male) probands had a two-fold higher mortality RR when the paternal grandmother experienced good availability during her SGP, compared to the mortality risk of probands whose paternal grandmothers had other experiences during the SGP $(P=0.001)$. Poor food supply during the SGPs of the paternal grandparents was followed by the opposite effect. The male probands whose paternal grandfather had such an experience had a reduced $(P=0.025)$ mortality RR of 0.65 and female probands had some tendency $(P=0.12)$ to reduced mortality RR $(0.72)$ when the paternal grandmother had poor food supply.

The 'criss-cross' paternal grandparental/grandchild effects, transmitted through the same set of fathers, creates a situation that is 'internally controlled' (see Discussion). Therefore, all subsequent analyses focus on the paternal grandparental/grandchild effects. The association of the paternal grandfather's food supply with his grandson's (but not granddaughter's) mortality RR and the paternal grandmother's food supply with her granddaughter's (but not grandson's) mortality RR is seen in both the 1890 and 1905 cohorts (Table 3).

\section{Developmental periods when transgenerational effects are triggered}

Sex-specific analysis of the transgenerational outcomes through the father provides a means to explore the exposure-sensitive period(s) in the paternal grandparents in a controlled and rigorous manner. The result of bivariate analysis of the effect of their food availability at ages from conception to 20 years on the mortality RR of the probands is shown in Figure 1. Figure 1a reveals, with respect to paternal grandfathers/grandsons and paternal grand- mothers/granddaughters, three periods of exposure sensitivity for both grandparents with one period corresponding in time to the earlier derived SGP (see Materials and methods). All show remarkable sex specificity of outcome because, by contrast, Figure $1 \mathrm{~b}$ shows no transgenerational effect whatsoever with respect to paternal grandfathers/ granddaughters and paternal grandmothers/grandsons. When the transgenerational responses to 'good' and 'poor' food supply show a reciprocal pattern this implies a dose response across the range of grandparental food availability. Several intriguing features should be noted. There is a reversal in the direction of the association between the proband's mortality RR and the paternal grandparental food availability during the SGP, both for the paternal grandfather and the paternal grandmother. Food supply during the period from paternal grandmother's conception to age 3 years is associated with the largest transgenerational response. However, equally important, is no discernible transgenerational effect of exposure during puberty of either paternal grandparent.

\section{Discussion}

We have studied two general populations, a contemporary one (ALSPAC) with parental history taken before the birth of the study child followed by prospective collection of data, and a three-generation population (Överkalix) analysed using historical records. Our earlier Överkalix observations linking the paternal ancestors' food supply (specifically during their SGP in mid childhood) to offspring and grandchild mortality rate ${ }^{9,10}$ led us to test in ALSPAC the specificity of exposure in the SGP for triggering a transgenerational effect. Self-reported onset of paternal 

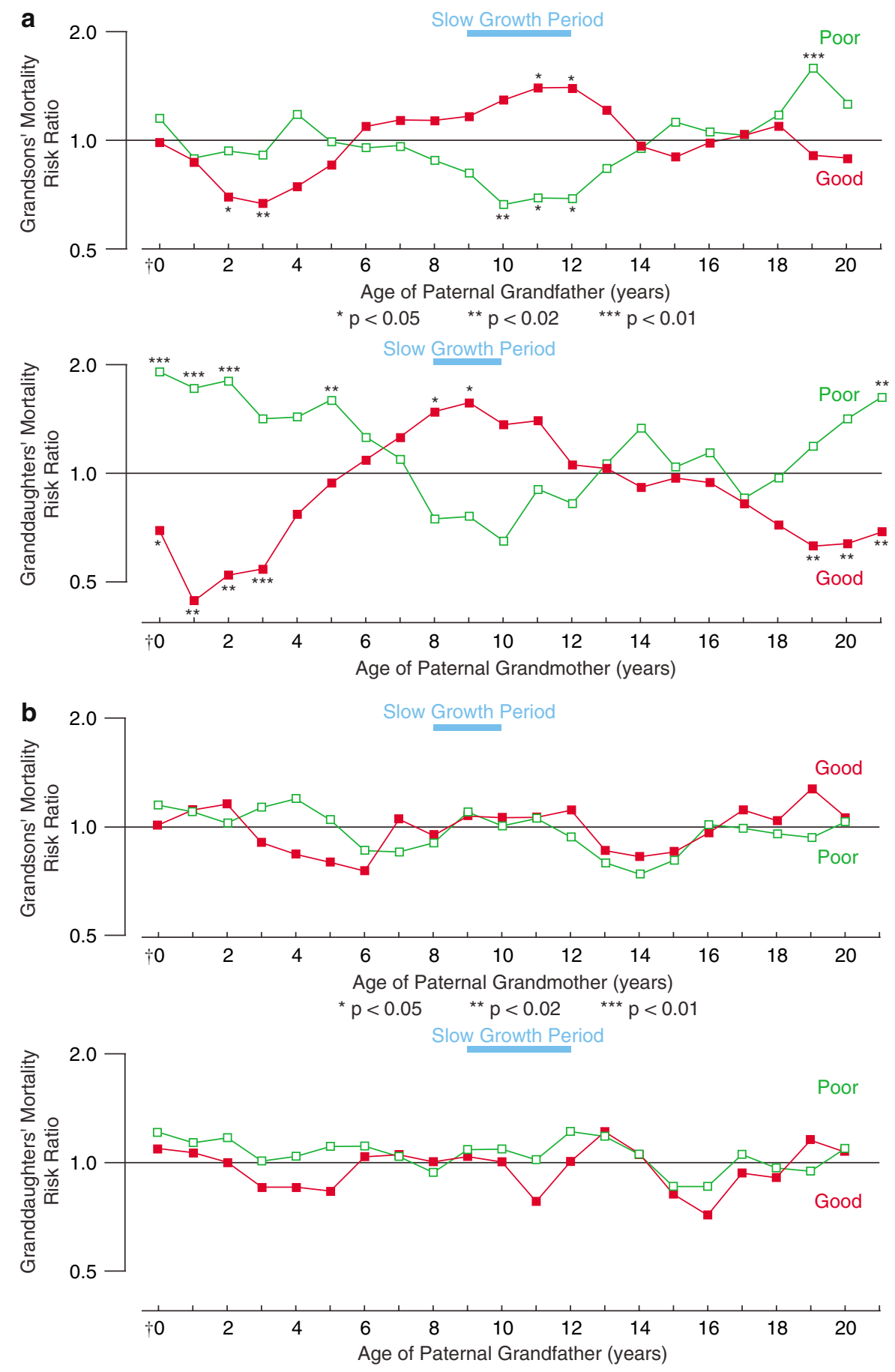

Figure 1 The effect of paternal grandparental food supply (good = red filled squares, poor = green open squares) at different times in their early life on the mortality rate of their grandchildren. Both ( $\mathbf{a}$ and $\mathbf{b}$ ) show on the Y-axis the mortality RR of the grandchildren separated by sex, first the grandsons' mortality RR and below this, the granddaughters' mortality RR. The age at which the paternal grandparent was exposed to good or poor food supply is given along the X-axis. The grandchildren's mean mortality RR results are plotted for both those paternal grandparents who had a good food supply (red) and those who had a poor supply (green) at the specified age on the X-axis. (a) First relates paternal grandfathers' exposure to his grandsons' mortality RR and then below the paternal grandmothers' exposure to her granddaughters' mortality RR. (b) First relates paternal grandmothers' exposure to his grandsons' mortality RR and then below the paternal grandfathers' exposure to her granddaughters' mortality RR. The data points were obtained using a 3-year frame, advanced one year at a time, for grandparental age at exposure, to produce rolling means for the grandchild's (proband's) mortality RR for both 'good' and 'poor' ancestral food supply. Exposure to at least 1 year of surfeit of food or to at least 1 year of poor availability during a 3-year period denotes it as exposure to a 'good' period or a 'poor' period respectively. "Food supply at age 0 years is the mean for the 33-month period from -267 days until the day before the second birthday and therefore includes fetal life. 
smoking was the available exposure with which we could explore the difference between exposure in the SGP and in later, pubertal stages of development. The choice of outcome in the ALSPAC child, namely growth with BMI as an exemplar, was based on what might be relevant to the observed Överkalix transgenerational effects on cardiovascular and diabetic deaths. ${ }^{10}$ Results in each population generated hypotheses that were then tested in the other, with the sex-specificity noted first in ALSPAC and then tested in the Överkalix cohorts.

In the present study we have observed the following:

(i) A transgenerational effect of father's mid-childhood smoking on BMI in his child at 9 years. We do not know the mid-childhood growth patterns of the ALSPAC fathers, but interpret the significant trend of decreasing BMI in the child with later onset of paternal smoking (Table 1) as reflecting the increasing proportion of fathers who had progressed from their SGP into the prepubertal growth spurt and puberty. This supports the Överkalix observation that exposure in the SGP, but not later in puberty, can lead to a transgenerational effect.

(ii) When adjusted for continued paternal smoking in order to reveal the effect of the age of his childhood onset of smoking per se, the transgenerational effect on $\mathrm{BMI}$ is restricted to boys.

(iii) Analysis of the three-generation Överkalix data by sex of the proband shows striking sex-specific effects (Table 2, Figures 1a) whereby the paternal grandfather's food supply was only linked to the mortality RR of grandsons, while paternal grandmother's food supply was only associated with the granddaughters' mortality RR. The absence of any association in the reverse paternal grandparent/grandchild pairings (Figure 1b) provides an important internal control for paternal-line social economic confounders, as the presence and absence of the transgenerational effect involves transmission through the same fathers.

(iv) These specific paternal grandparent/grandchild effects were seen in two completely separate cohorts, 1890 and 1905. The almost absent effect in the 1920 cohort could be due to their ancestors experiencing less extreme changes in food supply.

(v) The clear pattern of specific, limited 'exposure-sensitive' periods throughout the paternal grandparents' development from conception to adulthood (Figure 1a), including a switch in the direction of the transgenerational association during the SGP, confirms the importance of the SGP and suggests the existence of an evolved transgenerational response mechanism.

Despite the difficulties with such observational research (discussed below), we believe that together the ALSPAC and
Överkalix results provide proof of principle that a sexspecific, male-line transgenerational response system exists in humans. Our data suggest that it is triggered by exposures at very specific times during the ancestors' development, and the finding that the transgenerational effect is sex-specific represents the first step in defining the underlying mechanism.

Animal experiments have demonstrated that a particular exposure in the male can result in a specific phenotypic change in subsequent generations. ${ }^{7,8,16-19}$ Little is known about the transmitting mechanisms, except in the specific study of epigenetic states at the Axin ${ }^{F u}$ allele, ${ }^{7}$ and the altered DNA methylation patterns at two loci in the epididymal sperm of rats with reduced spermatogenic capacity due to their paternal ancestor being exposed (in utero) to the endocrine disruptor vinclozolin. ${ }^{8}$ In rats paternal dexamethasone exposure in utero led to both a reduced birth weight and increased hepatic activity of the gluconeogenic enzyme, phosphoenolpyruvate carboxykinase, in future offspring. ${ }^{19}$ Beyond studies of mutagenic agents, ${ }^{20}$ we are not aware of any general male-line transgenerational studies in humans. This probably stems from two factors; residual scepticism ${ }^{21}$ about the germline's susceptibility to environmentally-induced change and epigenetic inheritance (with its hint of Lamarkism), and the fact that human transgenerational studies are fraught with problems.

Three-generation historical records are necessarily incomplete. In the Överkalix analysis we have deliberately focused on the paternal line and particularly the influence of grandparental food supply on the mortality rate of the grandchild (proband). However, despite the fortuitous paternal-line 'internal control' provided by the sex-specific nature of the transgenerational associations (Figure 1b), we do not present data adjusted for the probands' own childhood circumstances that are likely to affect longevity. $^{22}$ These analyses are underway (Kaati et al, unpublished observations) and show that the same sex-specific grandparent/grandchild associations remain significant, if slightly attenuated. What is changed is the effect of the father's food supply on offspring mortality rate. Unadjusted (Table 2), the association is with his daughter's mortality rate, but after adjustment for the proband's childhood circumstances (father's landownership and occupation, the maintenance of the nuclear family, and the parents level of literacy) a male-specific effect emerges (fathers' good food supply in his SGP being associated with increased mortality rate in just sons RR $1.68, P=0.01$ ).

The comprehensive nature of ALSPAC does allow appropriate statistical control for confounders with respect to associations between age of paternal onset of smoking and the selected outcomes. These outcomes were strictly limited to the prior hypothesis of relevance to early risk factors for cardiovascular and diabetic death, namely, birth weight and gestation length, and height, weight, blood 
pressure and cholesterol at 7 and/or 9 years. After appropriate adjustment a significant association was also found with gestation length, again limited to boys (data not shown; Golding, personal communication). It is theoretically possible (and also testable in the future) that the ALSPAC results could be due to a pleiotropic gene effect that predisposes fathers to start smoking in mid childhood (but not later) and then, if transmitted to a son, also predisposes to a high BMI. However, the coherence between the ALSPAC and Överkalix results in terms of the exposure-sensitive periods and sex specificity supports the hypothesis that there is a general mechanism for transmitting information about the ancestral environment down the male line.

In terms of the ancestral environment in Överkalix, we have no record of actual nutrition and have to use food availability as a surrogate estimate. In both populations, the exposures used in the analysis may not be directly causal but strongly associated with whatever it is that triggers the transgenerational effect.

In considering the sex-specific nature of the associations in the Överkalix data, it is noteworthy that we are not dealing with sex-limitation (a widespread phenomenon); the effect sizes are comparable in grandsons and granddaughters (Tables 2 and 3, Figure 1a). In the ALSPAC data, sex-limitation remains a possible explanation for why only sons show transgenerational associations with paternal mid-childhood onset of smoking; the mediating mechanism, whether it be genetic, epigenetic or some other, could be affecting testosterone-sensitive pathways only. However, if the ALSPAC and Överkalix observations do stem from a common underlying mechanism for capturing environmental information during specific developmental periods and transmitting it to subsequent generations, then what could explain the curious 'criss-cross' sexspecific patterns of transmission of the observed transgenerational effects; paternal grandfather to just grandsons and paternal grandmother to just granddaughters (Table 3)? We think the most parsimonious hypothesis is a role for the sex chromosomes, $\mathrm{X}$ and Y. Father to just son (as in ALSPAC) and paternal grandfather to just grandson effects could be mediated (at least in part) by something carried on the $\mathrm{Y}$, while the $\mathrm{X}$ chromosome transmitted by a woman to her son can only be passed on to her granddaughter, not grandson. This hypothesis does not preclude downstream involvement of autosomal genes.

Another intriguing aspect of the sex-specificity observed is the timing of the exposure-sensitive periods during the development of the paternal grandparents (Figure 1a). In speculating that the environment might be able to modify germline epigenetic imprints at specific stages in gametogenesis, ${ }^{23}$ one might expect different periods of exposure sensitivity between males and females, since the main period of oogenesis occurs in fetal life unlike spermatogenesis. While extremes of food supply in the SGP of both paternal grandparents were associated with trangenerational effects, the major exposure sensitive period detected in the paternal grandmother was when she was a fetus/ infant. With respect to the paternal grandmother's SGP, it is perhaps relevant that recent evidence from mice show that oogenesis continues after birth. ${ }^{24}$ In both paternal grandparents the direction of the transgenerational association with grandchild mortality reverses for exposure during the grandparents' mid childhood, a time that corresponds exactly to the SGP derived earlier from published growth velocity data (as indicated by bars in Figure 2). ${ }^{9,13,14}$ This suggests the SGP is a 'critical' ontogenetic period, but why the transgenerational response reverses is unclear.

The possible involvement of the $\mathrm{Y}$ chromosome was unexpected. Genomewide association studies tend not to include Y markers, but one study of exceptional longevity with preserved cognition found an association with Yq markers close to the centromere, in addition to the expected association with the APOE epsilon2 allele. ${ }^{25}$

Our findings add a new, multigenerational dimension to the interplay between inheritance and environment in health and development; they provide proof of principle that sex-specific, male-line transgenerational effects exist in humans. We propose that our results, which are specific enough to allow replication, are manifestations of an evolved adaptive transgenerational response mechanism. Our study exemplifies a research approach that could, potentially, make a major contribution to public health and impact on the way we view our responsibilities towards future generations.

\section{Acknowledgements}

We are extremely grateful to all the mothers and their partners who took part and to the midwives for their cooperation and help in recruitment into ALSPAC. The whole ALSPAC study team comprises interviewers, computer technicians, laboratory technicians, clerical workers, research scientists, volunteers and managers who continue to make the study possible. ALSPAC is supported by the Wellcome Trust, the Medical Research Council (MRC), the Department of Health, the Department of the Environment and many others. We acknowledge Lennart Backman, MD, for his contribution to the Swedish study that was supported by the Swedish Councils for Planning and Coordination of Research (FRN 940020:4) and Working Life and Social Research (FAS 2001-1061). We thank Marilyn Monk and Tim Cole for comments on earlier manuscripts.

\section{References}

1 Drake AJ, Walker BR: The intergenerational effects of fetal programming: non-genomic mechanisms for the inheritance of low birth weight and cardiovascular risk. J Endocrinol 2004; 180: $1-16$.

2 Shorter J, Lindquist S: Prions as adaptive conduits of memory and inheritance. Nature Rev Genet 2005; 6: 435-450.

3 Ostermeier GC, Miller D, Huntriss JD, Diamond MP, Krawetz S Delivering spermatozoan RNA to the oocyte. Nature 2004; 429: 154 . 
4 Yasui K, Mihara S, Zhao C et al: Alteration in copy numbers of genes as a mechanism for acquired drug resistance. Cancer Res 2004; 64: $1403-1410$.

5 Epel ES, Blackburn EH, Lin J et al: Accelerated telomere shortening in response to life stress. Proc Natl Acad Sci 2004; 101: $17312-17315$.

6 Bird A: DNA methylation patterns and epigenetic memory. Genes Dev 2002; 16: 6-21.

7 Rakyan VK, Chong S, Champ ME et al: Transgenerational inheritance of epigenetic states at the murine $\mathrm{Axin}^{\mathrm{Fu}}$ allele occurs following maternal and paternal transmission. Proc Natl Acad Sci 2003; 100: 2538-2543.

8 Anway MD, Cupp AS, Uzumcu M, Skinner MK: Epigenetic transgenerational actions of endocrine disruptors and male fertility. Science 2005; 308: 1466-1469.

9 Bygren LO, Kaati G, Edvinsson S: Longevity determined by ancestors' overnutrition during their slow growth period. Acta Biotheoretica 2001; 49: 53-59.

10 Kaati G, Bygren LO, Edvinsson S: Cardiovascular and diabetes mortality determined by nutrition during parents' and grandparents' slow growth period. Eur J Hum Genet 2002; 10: 682-688.

11 Golding J, Pembrey M, Jones R, ALSPAC Study Team: ALSPAC the avon longitudinal study of parents and children. I. Study methodology. Paediatr Perinat Epidemiol 2001; 15: 74-87.

12 Ness AR: The avon longitudinal study of parents and children (ALSPAC) - a resource for the study of the environmental determinants of childhood obesity. Eur J Endocrinol 2004; 151: U141-U149.

13 Prader A, Largo RH, Molinari L, Issler C: Physical growth of Swiss children from birth to 20 years of age. First Zurich Study of Growth and Development. Helv Paediatr Acta 1989; 43 (Suppl 52): $1-125$.

14 Tanner JM: A history of the study of human growth. Cambridge: Cambridge University Press, 1981.
15 SAS/STAT software, Version 8.2 of SAS System for Windows. Copyright 1999-2001 Cary, NC, USA: SAS Institute Inc.

16 Campbell JH, Perkins P: Transgenerational effects of drug and hormonal treatments in mammals: a review of observations and ideas. Prog Brain Res 1988; 73: 535-553.

17 Boucher BJ, Ewen SW, Stowers JM: Betel nut (Areca catechu) consumption and the induction of glucose intolerance in adult CD1 mice and in their F1 and F2 offspring. Diabetologia 1994; 37: $49-55$.

18 Barber R, Plumb MA, Boulton E, Roux I, Dubrova Y: Elevated mutation rates in the germ line of first- and second-generation offspring of irradiated male mice. Proc Natl Acad Sci 2002; 99: 6877-6882.

19 Drake AJ, Walker BR, Seckl JR: Intergenerational consequences of fetal programming by in utero exposure to glucocorticoids in rats. Am J Physiol Regul Integr Comp Physiol 2005; 288: R34-R38.

20 Robaire B, Hales BF (eds): Advances in Male Mediated Developmental Toxicity. New York: Kluwer Academic/Plenum Publishers, 2003.

21 Weismann A: The Germ-Plasm: A Theory of Heredity, (translated from the 1892 German edn by W Newton and H Ronnfeldt). London: Walter Scott, 1893.

22 Graham H, Power C: Childhood disadvantage and health inequalities: a framework for policy based on lifecourse research. Child Care Health Dev 2004; 30: 671-678.

23 Pembrey ME: Time to take epigenetic inheritance seriously. Eur J Hum Genet 2002; 10: 669-671.

24 Johnson J, Canning J, KanekoT et al.: Germline stem cells and follicular renewal in the postnatal mammalian ovary. Nature 2004; 428: 145 - 150 .

25 Zubenko GS, Stiffler JS, Hughes, Fatigati MJ, Zubenko WN: Genome survey for loci that influence successful aging: sample characterization, method validation, and initial results for the Y chromosome. Am J Geriatr Psychiatry 2002; 10: 619-630. 